\title{
Gastroenterologists' preference and risk perception on the use of immunomodulators and biological therapies in elderly patients with ulcerative colitis: an international survey
}

\author{
Webber Chan ${ }^{a, b, \star}$, Viraj C. Kariyawasam ${ }^{a, c, \star}$, Shin Kim ${ }^{d}$, Aviv V. Pudipeddia, \\ Sudarshan Paramsothy ${ }^{a}$, Hang Hock Shim ${ }^{b}$, Fadi H. Mourad ${ }^{a}$, Nik Ding ${ }^{e}$, Marc Ferrante ${ }^{f}$ and \\ Rupert W. Leonga,d
}

Background and aims: Comorbidities, polypharmacy, malignancies, and infections complicate management of elderly patients with inflammatory bowel diseases (IBD). This study assessed gastroenterologists' preference in the prescription of medications or surgery to elderly patients with IBD, and the factors associated with their choices.

Methods An international case-based survey was conducted that presented three cases of steroid-dependent ulcerative colitis assessing young-age versus elderly-age patients, with and without comorbidity. Physician characteristics and practice demographics were collected. Factors associated with selection of different choices of therapy were determined by logistic regression analysis.

Results A total of 424 respondents from 41 countries were included. Vedolizumab (53.2\%) and thiopurines (19.4\%) were the top treatment preferences for moderate-to-severe ulcerative colitis $(P<0.0001)$. Comorbidity and older age were independently associated with more frequent use of vedolizumab $(P<0.0001)$, and less frequent use of immunomodulators and anti-tumour necrosis factor (TNF; $P<0.0001)$. Comorbidity was the only independent predictor for selecting colectomy $(P<0.0001)$. A history of lymphoma $(94 \%)$ and opportunistic infection $(78.3 \%)$ were the most frequent conditions precluding the use of thiopurine and anti-TNF in elderly patients with IBD. Only $6.1 \%$ of respondents considered patient age a limit for vedolizumab, while $37.9 \%$ considered age as a limiting factor in prescribing thiopurines $(P<0.001)$. Geographical heterogeneity was identified with significantly more physicians from Oceania and North America favouring the use of vedolizumab.

Conclusion Vedolizumab was the preferred first-line agent in the treatment of elderly patients with IBD with steroiddependent moderate-to-severe ulcerative colitis. Older age and presence of comorbidity influenced the selection of medication. Comorbidity was the main predictor of colectomy. Geographical heterogeneity in prescribing habits may relate to medication reimbursement in individual countries. Eur J Gastroenterol Hepatol XXX: 00-00

Copyright @ 2020 Wolters Kluwer Health, Inc. All rights reserved.

\section{Introduction}

Inflammatory bowel disease (IBD), comprising Crohn's disease and ulcerative colitis, is a global disease with

\footnotetext{
European Journal of Gastroenterology \& Hepatology 2020, XXX:00-00

Keywords: colectomy, elderly, inflammatory bowel disease, ulcerative colitis, vedolizumab

${ }^{a}$ Gastroenterology and Liver Services, Concord Repatriation General Hospital, Sydney, New South Wales, Australia, 'bepartment of Gastroenterology, Singapore General Hospital, Singapore, 'Faculty of Medicine, University of Sydney, dFaculty of Medicine, The University of New South Wales, Sydney, eDepartment of Gastroenterology, St Vincent's Hospital, Melbourne, Australia and 'Department of Gastroenterology, University Hospitals Leuven, KU Leuven, Leuven, Belgium

Correspondence to Webber Chan, MRCP, Gastroenterology and Liver Services, Concord Repatriation General Hospital, Sydney, New South Wales, Australia E-mail: webber.chan.p.w@singhealth.com.sg

${ }^{\star}$ Dr. Webber Chan and Dr. Viraj C. Kariyawasam contributed equally to the writing of this article.

Received 21 October 2019 Accepted 13 January 2020

Supplemental Digital Content is available for this article. Direct URL citations appear in the printed text and are provided in the HTML and PDF versions of this article on the journal's website, www.eurojgh.com
}

increasing incidence and prevalence [1]. The rising incidence has been reported across all age groups including the elderly population. Elderly patients with IBD include those diagnosed at a younger age who transition to an elderly age and those diagnosed after the age of 60 years (elderly-onset IBD) [2]. An estimated $10-30 \%$ of patients with IBD worldwide are aged $>60$ years [3], and recent studies have found that between 10 and $23 \%$ of patients are diagnosed with IBD after the age of 60 years [4,5]. As the global population ages, the prevalence of IBD in the elderly is also expected to rise, and peaks at 1061 per 100000 in patients $>85$ years old [6].

Conventional IBD therapy consists of mesalazine, corticosteroids, and immunomodulators which include thiopurines (such as azathioprine and 6-mercaptopurine) and methotrexate. Anti-tumour necrosis factor (TNF)- $\alpha$ agents (infliximab, adalimumab, certolizumab pegol, and golimumab), as well as anti-integrin agents (vedolizumab) [7] have been approved for induction and maintenance therapy in both moderate-to-severe Crohn's disease and ulcerative colitis. More recently, the anti-IL 12/23 agent ustekinumab [8] and tofacitinib, a nonselective janus 
kinase inhibitor [9], have been approved. Expert consensus suggests that the principles of medical management of elderly-onset IBD are generally the same as in other age groups [2]. However, a recent meta-analysis and systematic review have reported that elderly-onset IBD patients use less conventional immunomodulators and biological therapy, but have comparable or even higher rates of IBD-related surgery than younger-onset patients [10]. Moreover, corticosteroids are often prescribed to the elderly IBD patients, with $32 \%$ of patients with IBD aged $>65$ years maintained on steroids for $>6$ months [11]. It is uncertain if the identified differences in the utilisation of immunosuppressive therapies and surgery between young-onset and elderly-onset IBD reflect a more aggressive disease course in elderly-onset IBD, or reluctance of physicians to prescribe immunomodulators and biological therapies because of concern of adverse events precipitated by concurrent comorbidities [12], polypharmacy and potential for drug interactions [13], or medication nonadherence issues [14] in elderly subjects. Both thiopurine and anti-TNF uses are associated with increased risk of lymphoma [15], and serious and opportunistic infections [16-18]. Moreover, thiopurine use is associated with nonmelanoma skin cancer $[19,20]$. Efficacy and safety data of biological agents in elderly patients with IBD are scarce, as most pivotal clinical trials excluded the recruitment of elderly subjects.

The attending physicians' knowledge and risk perception towards the use of immunomodulators and biological agents in the elderly may influence prescription practice. Medication class preferences may differ worldwide due to different pharmaceutical or health insurance reimbursement schemes. Avoidance of medication cost or medication unavailability might drive the selection of colectomy over medical therapy. We, therefore, conducted an international survey to determine the preferences of gastroenterologists on prescribing medical treatment versus colectomy in elderly patients with steroid-dependent ulcerative colitis and to determine whether age alone, presence of comorbidities or both, may drive treatment preference. Secondary aims were to identify factors associated with treatment preference, identify conditions that preclude gastroenterologists from prescribing immunomodulators and biological agents, and determine whether patient age is considered a limitation to prescribing the various medication classes.

\section{Materials and methods}

\section{Survey design}

This investigator-initiated study was conducted in collaboration with two operational boards of the European Crohn's and Colitis Organisation (ECCO): Young-ECCO (Y-ECCO) and the clinical committee of ECCO (ClinCom). The decision to support this proposal was made by these committees through an independent review board following an international call to submit projects. The first version of the survey was designed by V.K., S.K. and R.L., and reviewed by Y-ECCO, ClinCom and the members of the ECCO governing board. After two rounds of modifications, the final version of the survey was approved by consensus. The survey consisted of 17 questions and was divided into four categories. First, demographic information, including medical position, continent and country of practice, years in gastroenterology practice, number of patients with IBD reviewed per week, and practice type was collected for each gastroenterologist. Second, we presented the clinical cases of three patients, both young and elderly, either with or without comorbidity, who had steroid-dependent ulcerative colitis. Third, we assessed the conditions that precluded gastroenterologists from prescribing the immunomodulators and biological agents. Fourth, the age limits of ordering such medications were recorded. The estimated time to complete the survey was 5 min. (Supplement 1, Supplemental digital content 1, http://links.lww.com/EJGH/A538). As this was a general survey and not developed as a quantitative tool, it was validated for semantics and understandability by testing on three gastroenterologists.

\section{Study population}

The inclusion criteria were adult gastroenterologists who completed $>50 \%$ of the questionnaire. In the absence of a universal definition of an 'IBD subspecialist' and in accord with previous studies [21], we pragmatically defined gastroenterologists as IBD subspecialists if they looked after $>100$ patients with IBD per year. The survey was distributed in hard copy format and through an online platform (SurveyMonkey) to gastroenterologist attendees at conferences and online mailing lists.

\section{The case scenarios}

Three case scenarios were designed to assess the impact of age, comorbidity, and the combination of both, on the choice of treatment modalities for ulcerative colitis. The first case scenario was a 76-year-old female with steroid-dependent ulcerative colitis, despite adherence to oral mesalazine $4 \mathrm{~g}$ daily. She required three courses of oral corticosteroids over a 9-month period (Supplement 1, Supplemental digital content 1, http://links.lww.com/EJGH/A538). Her colonoscopy revealed extensive colitis consistent with the Mayo endoscopic subscore of 3 . The patient had no other comorbidity (hereinafter referred to as 'elderly ulcerative colitis without comorbidity'). Scenario 2 was similar to the first one but the patient had comorbidities including type 2 diabetes mellitus with diabetic nephropathy and ductal adenocarcinoma of the left breast in remission ('elderly ulcerative colitis with comorbidity'). Scenario 3 was identical to scenario 2 but the patient was now 28 years old ('young ulcerative colitis with comorbidity').

All three case scenarios included five treatment options including prescribing immunomodulators, high-dose maintenance corticosteroids, mono- or combination therapy with anti-TNF, mono- or combination therapy with vedolizumab, and colectomy. The participants were asked to rank their choices of treatment, with one being the most preferred to five the least preferred.

\section{Statistical analysis}

Descriptive statistics of demographic data and overall responses for each question were documented. Categorical variables were expressed as frequencies and percentages, and continuous variables were expressed as medians 


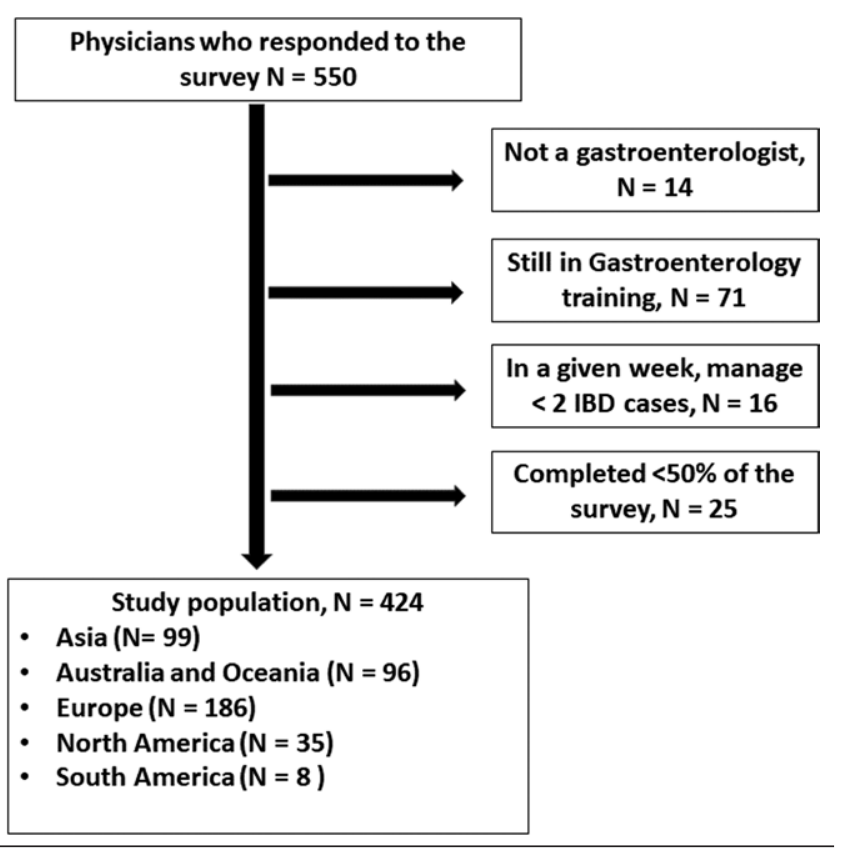

Fig. 1. Flow diagram showing the recruitment of gastroenterologists globally.

and interquartile range (IQR). Comparison of responses between groups was by chi-square test for categorical variables, Kruskal-Wallis test for nonparametrically distributed scales, and the ANOVA $t$-test for parametrically distributed scales, as appropriate. Univariate and multivariate binary logistic regression was used to determine variables associated with each treatment choice. Variables with a $P$-value of $<0.05$ in univariate analysis were included in multivariate analysis. Results were expressed as odds ratios (OR) and their 95\% confidence intervals (95\% CI). Statistical analyses were conducted using SPSS [IBM SPSS Statistics for Windows, version 23 (IBM Corp., Armonk, New York, USA)].

\section{Ethics}

The anonymous survey was approved by the Centralised Institutional Review Board (CIRB) of SingHealth Research (CIRB Reference number: 2019/2056) and completion and return of the survey were deemed informed consent. The study protocol conforms to the ethical guidelines of the 1975 Declaration of Helsinki (sixth revision, 2008) as reflected in a priori approval by the institution's human research committee.

\section{Results}

\section{Study cohort}

The questionnaire was distributed in 41 countries covering five continents. The written survey had a response rate of $55 \%$. The online survey encouraged distribution amongst gastroenterology colleagues to increase the sample size. The exact response rate, therefore, was undefined. About 950 email invitations were sent. A total of 550 gastroenterologists responded to the survey, of whom 424 met the inclusion criteria (Fig. 1). Characteristics of the gastroenterologists included are depicted in Table 1 (and Supplementary Table 2, Supplemental digital content 1,

\begin{tabular}{ll}
\hline \multicolumn{2}{l}{ Table 1. Gastroenterologists' demographic and clinical characteristics } \\
\hline Gastroenterologists' characteristics & $N=424(\%)$ \\
\hline Continents & \\
Asia & Total $=99(23.3 \%)$ \\
Australia and Oceania & Total $=96(22.6 \%)$ \\
Europe & Total $=186(43.9 \%)$ \\
North America & Total $=35(8.3 \%)$ \\
South America & Total $=8(1.9 \%)$ \\
Years in practice & \\
Mean [years (SD)] & $13.9(9.7)$ \\
$<5$ years & $98(23.1)$ \\
$6-10$ years & $77(18.2)$ \\
11-15years & $60(14.2)$ \\
$>15$ years & $152(35.8)$ \\
Missing & $37(8.7)$ \\
Number of patients with IBD seen per week & \\
2-5 patients & $78(18.6)$ \\
$6-10$ patients & $59(13.9)$ \\
$>10$ patients & $286(67.5)$ \\
Practice setting & \\
Public & $291(68.6)$ \\
Private & $56(13.2)$ \\
Mixed (public + private) & $76(17.9)$ \\
Missing & $1(0.2)$
\end{tabular}

IBD, inflammatory bowel diseases.

http://links.lww.com/EJGH/A538). Of those surveyed, $43.9 \%(n=186)$ were from Europe, $23.3 \%(n=99)$ from Asia, and $22.6 \%(n=96)$ from Oceania. The median duration of gastroenterology experience was 12.0 years (IQR: $5.0-20.75$ ) with $69 \%$ working in public hospitals and $68 \%$ of respondents treating $>10$ patients with IBD per week.

\section{Response to case scenarios}

Vedolizumab was the most preferred treatment choice in all three cases, with $43.9,64.9$, and $50.9 \%$ of gastroenterologists selecting it as the first choice for cases 1,2 , and 3 , respectively (Table 2 ). In the 'elderly ulcerative colitis without comorbidity' (case 1) and 'young ulcerative colitis with comorbidity' (case 3), the second preferred option was immunomodulators (28.5 and $18.7 \%$, respectively), followed by anti-TNF (23.8 and $18.3 \%$, respectively). In the 'elderly ulcerative colitis with comorbidity' (case 2), colectomy was the second most preferred choice $(13.3 \%)$, followed by immunomodulators $(10.9 \%)$. Increasing the dose of prednisolone was the least preferred option among all 3 scenarios (Supplementary Table 3, Supplemental digital content 1, http://links.lww.com/EJGH/A538).

\section{Factors associated with treatment choices}

On multivariate analysis, comorbidity and older age were independent factors associated with less frequent use of immunomodulators. Compared with 'elderly ulcerative colitis with comorbidity' (case 2), immunomodulators were more frequently used in 'elderly ulcerative colitis without comorbidity' (case 1; OR, 4.06; 95\% CI, $2.26-6.29 ; P<0.0001)$ and 'young ulcerative colitis with comorbidity' (case 3; OR, 2.28; 95\% CI, 1.45-3.58; $P<0.0001$; Table 4). Other factors associated with less frequent prescription of immunomodulators included practicing in Europe (OR, 0.51; 95\% CI, 0.34-0.79; $P=0.002$ ) and North America (OR, 0.03; 95\% CI, 0.004-0.22; $P=0.001)$ compared to Asia; having a perceived maximum age limit to prescribing thiopurines $(\mathrm{OR}, 0.46 ; 95 \% \mathrm{CI}$, 
Table 2. First choices of treatment based on case scenarios

Case scenarios

\begin{tabular}{|c|c|c|c|c|c|}
\hline First choice of treatment & $\begin{array}{c}\text { Elderly ulcerative colitis } \\
\text { without comorbidity (case 1) }\end{array}$ & $\begin{array}{l}\text { Elderly ulcerative colitis } \\
\text { with comorbidity (case 2) }\end{array}$ & $\begin{array}{l}\text { Young ulcerative colitis } \\
\text { with comorbidity (case 3) }\end{array}$ & Overall (\%) & $P$ value \\
\hline Prescribe immunomodulators & $28.5 \%(113 / 397)$ & $10.9 \%(43 / 394)$ & $18.7 \%(73 / 390)$ & 19.4 & $P<0.0001$ \\
\hline $\begin{array}{l}\text { Increase dose of prednisolone and } \\
\text { continue on that dose for maintenance }\end{array}$ & $1.8 \%(7 / 397)$ & $3.0 \%(12 / 395)$ & $2.1 \%(8 / 389)$ & 2.7 & $P=0.622$ \\
\hline Prescribe anti-TNF & $23.8 \%(95 / 400)$ & $8.8 \%(35 / 396)$ & $18.3 \%(72 / 394)$ & 17.0 & $P<0.0001$ \\
\hline Prescribe vedolizumab & $43.9 \%(175 / 399)$ & $64.9 \%(259 / 399)$ & $50.9 \%(200 / 393)$ & 53.2 & $P<0.0001$ \\
\hline Recommend colectomy & $2.8 \%(11 / 397)$ & $13.3 \%(53 / 399)$ & $11.5 \%(45 / 393)$ & 9.2 & $P<0.0001$ \\
\hline
\end{tabular}

TNF, tumour necrosis factor.

Table 3. Conditions precluding the use of immunomodulators and biological agents in elderly patients with inflammatory bowel diseases

Preclude commencing medications (\% from respondents within the drug)

\begin{tabular}{|c|c|c|c|c|c|}
\hline Conditions & Missing input (\% of total) & Immuno-modulator & Anti-TNF & Vedolizumab & $P$ value \\
\hline History of lymphoma & 16 & 94.0 & 69.0 & 30.4 & $<0.0001$ \\
\hline History of solid organ cancer & 5.7 & 65.1 & 57.4 & 20.0 & $<0.0001$ \\
\hline Melanoma skin cancer & 16.3 & 44.7 & 61.0 & 20.6 & $<0.0001$ \\
\hline Nonmelanoma skin cancer & 14.3 & 53.2 & 29.2 & 13.6 & $<0.0001$ \\
\hline Congestive heart failure & 16.1 & 5.3 & 76.6 & 12.1 & $<0.0001$ \\
\hline Demyelination & 14.2 & 7.8 & 61.8 & 19.5 & $<0.0001$ \\
\hline Osteoporosis & 16.3 & 1.5 & 1.0 & 0.7 & 0.627 \\
\hline Hepatotoxicity & 5.8 & 69.1 & 22.2 & 19.1 & $<0.0001$ \\
\hline Opportunistic infection & 16.2 & 72.6 & 78.3 & 57.4 & $<0.0001$ \\
\hline Drug to drug interaction & 14.5 & 52.3 & 31.4 & 38.2 & $<0.0001$ \\
\hline
\end{tabular}

TNF, tumour necrosis factor.

$0.29-0.68 ; P<0.001$ ), steroids (OR, 0.32; 95\% CI, 0.13$0.79 ; P=0.014$ ) and combination therapy (OR, $0.64 ; 95 \%$ CI, 0.43-0.96; $P=0.029)$. In contrast, working in private practice (OR 1.69; 95\% CI, 1.05-2.73; $P=0.03$ ); and having a perceived age limit to prescribing an anti-TNF (OR, $1.73 ; 95 \%$ CI, 1.04-2.88; $P=0.036)$, and vedolizumab (OR, 3.45; 95\% CI, 1.76-6.76; $P<0.0001$ ) were associated with more frequent use of immunomodulators.

Similarly, the presence of comorbidity and older age were independently associated with less frequent use of anti-TNF. Compared with 'elderly ulcerative colitis with comorbidity' (case 2), anti-TNF was more frequently prescribed in 'elderly ulcerative colitis without comorbidity' (case 1) (OR, 3.57; 95\% CI, 2.21-5.77; $P<0.0001)$ and 'young ulcerative colitis with comorbidity' (case 3) (OR, 2.50; 95\% CI, 1.53-4.10; $P<0.0001$ ) (Table 4). Other factors associated with more frequent prescription of antiTNF was practicing in South America (OR, 4.29; 95\% CI, 1.45-12.69; $P=0.009)$ compared to Asia, and having a perceived age limit in prescribing corticosteroids (OR, $1.95 ; 95 \% \mathrm{CI}, 1.01-3.78 ; P=0.048)$. Increased years practicing in gastroenterology and seeing $>10$ patients with IBD per week, were associated with less frequent use of anti-TNF (OR, 0.80; 95\% CI, 0.70-0.93; $P=0.003$, and OR, 0.52; 95\% CI, 0.32-0.82; $P=0.005$, respectively).

On multivariate analysis, comorbidity and old age were independently associated with more frequent use of vedolizumab. Vedolizumab was less frequently used in 'elderly ulcerative colitis without comorbidity' (case 1) (OR, 0.37; 95\% CI, 0.27-0.51; $P<0.0001$ ) and 'young ulcerative colitis with comorbidity' (case 3) (OR, 0.51; 95\% CI, $0.37-0.69 ; P<0.0001)$ compared against 'elderly ulcerative colitis with comorbidity' (case 2; Table 4). Other factors associated with more frequent prescription of vedolizumab included practicing in Oceania and North
America (OR, 1.47; 95\% CI, 1.02-2.13; $P=0.04$ and OR, 5.58; 95\% CI, 2.96-10.51; $P<0.0001$, respectively) compared to Asia; seeing $>10$ patients with IBD per week (OR, 1.92; 95\% CI, 1.36-2.71; $P<0.0001)$, and having a perceived age limit in prescribing thiopurines $(\mathrm{OR}, 1.54$; 95\% CI, 1.17-2.03; $P=0.002$ ).

The presence of comorbidity was the only independent predictor for selecting colectomy. Compared with 'elderly ulcerative colitis with comorbidity' (case 2), surgery was less frequently recommended in 'elderly ulcerative colitis without comorbidity' (case 1) (OR, 0.18; 95\% CI, 0.09$0.36 ; P<0.0001)$. There was no statistically significant difference in recommending colectomy between 'elderly ulcerative colitis with comorbidity' and 'young ulcerative colitis with comorbidity' (OR, 0.81; 95\% CI, 0.52-1.26; $P=0.34)$. Corticosteroid use was not associated with age or comorbidity (Supplementary Table 4, Supplemental digital content 1, http://links.lww.com/EJGH/A538).

\section{Conditions precluding immunomodulators and biological agents in elderly patients with inflammatory bowel diseases}

A history of lymphoma $(94.0 \%)$ and opportunistic infection $(78.3 \%)$ were the commonest reported events precluding the use of thiopurines and anti-TNF in elderly patients with IBD (Table 3). Opportunistic infection $(57.4 \%)$ was the commonest condition considered as contraindications to vedolizumab in elderly patients with IBD.

\section{Age limit for prescribing immunomodulators and biological therapy}

Vedolizumab and corticosteroids were prescribed without age as a limiting factor by 93.9 and $93.7 \%$ of respondents, respectively. However, $37.9 \%$ of gastroenterologists 


\begin{tabular}{|c|c|c|c|c|c|c|c|c|}
\hline \multirow[b]{2}{*}{ Covariant considered in univariate analysis } & \multicolumn{2}{|c|}{ Immunomodulators } & \multicolumn{2}{|l|}{ Anti-TNF } & \multicolumn{2}{|c|}{ Vedolizumab } & \multicolumn{2}{|l|}{ Surgery } \\
\hline & OR $(95 \% \mathrm{Cl})$ & $P$ & OR $(95 \% \mathrm{Cl})$ & $P$ & OR $(95 \% \mathrm{Cl})$ & $P$ & OR $(95 \% \mathrm{Cl})$ & $P$ \\
\hline \multicolumn{9}{|l|}{ Scenario } \\
\hline Scenario 2 (reference) & 1 & & 1 & & 1 & & 1 & \\
\hline Scenario 1 (vs. 2) & $4.06(2.26-6.29)$ & $<0.0001$ & $3.57(2.21-5.77)$ & $<0.0001$ & $0.37(0.27-0.51)$ & $<0.0001$ & $0.18(0.09-0.36)$ & $<0.0001$ \\
\hline Scenario 3 (vs. 2) & $2.28(1.45-3.58)$ & $<0.0001$ & $2.50(1.53-4.10)$ & $<0.0001$ & $0.51(0.37-0.69)$ & $<0.0001$ & $0.81(0.52-1.26)$ & 0.34 \\
\hline \multicolumn{9}{|l|}{ Continents } \\
\hline Asia & 1 & & 1 & & 1 & & 1 & \\
\hline Oceania & $1.12(0.71-1.76)$ & 0.55 & $0.72(0.41-1.26)$ & 0.26 & $1.47(1.02-2.13)$ & 0.04 & $0.53(0.25-1.10)$ & 0.09 \\
\hline Europe & $0.51(0.34-0.79)$ & 0.002 & $1.51(0.95-2.39)$ & 0.08 & $1.22(0.89-1.68)$ & 0.22 & $1.43(0.86-2.34)$ & 0.17 \\
\hline North America & $0.03(0.004-0.022)$ & 0.001 & $0.68(0.29-1.57)$ & 0.36 & $5.58(2.96-10.51)$ & $<0.0001$ & $0.56(0.21-1.54)$ & 0.26 \\
\hline South America & $0.26(0.03-2.02)$ & 0.20 & $4.29(1.45-12.69)$ & 0.009 & $0.56(0.20-1.55)$ & 0.27 & $0.41(0.05-3.48)$ & 0.41 \\
\hline Years as gastroenterologist (years) & * & & $0.80(0.70-0.93)$ & 0.003 & * & & * & \\
\hline Number of IBD patients reviewed per week & * & & & & & & * & \\
\hline $2-5$ & & & 1 & & 1 & & & \\
\hline $6-10$ & & & $0.77(0.43-1.39)$ & 0.38 & $1.08(0.69-1.67)$ & 0.74 & & \\
\hline$>10$ & & & $0.52(0.32-0.82)$ & 0.005 & $1.92(1.36-2.71)$ & $<0.0001$ & & \\
\hline Place of practice & & & * & & * & & & \\
\hline Public & 1 & & & & & & 1 & \\
\hline Private & $1.69(1.05-2.73)$ & 0.03 & & & & & $0.46(0.20-1.07)$ & 0.07 \\
\hline Mixed & $1.25(0.81-1.04)$ & 0.30 & & & & & $0.51(0.26-1.01)$ & 0.052 \\
\hline Age limit for thiopurines & $0.46(0.29-0.68)$ & $<0.0001$ & * & & $1.54(1.17-2.03)$ & 0.002 & * & \\
\hline Age limit for vedolizumab & $3.45(1.76-6.76)$ & $<0.0001$ & * & & $0.23(0.11-0.48)$ & $<0.0001$ & $2.95(1.38-6.34)$ & 0.001 \\
\hline Age limit for anti-TNF & $1.73(1.04-2.88)$ & 0.036 & * & & $0.71(0.49-1.03)$ & 0.074 & $1.65(0.98-2.78)$ & 0.06 \\
\hline Age limit for steroids & $0.32(0.13-0.79)$ & 0.014 & $1.95(1.01-3.78)$ & 0.048 & * & * & * & \\
\hline Age limit for combination therapy & $0.64(0.43-0.96)$ & 0.029 & * & & * & & * & \\
\hline
\end{tabular}

Scenario 1: elderly ulcerative colitis only. Scenario 2: elderly ulcerative colitis with comorbidity. Scenario 3: young ulcerative colitis with comorbidity.

TNF, tumour necrosis factor; IBD, inflammatory bowel diseases.

*Factors not statistically significant on univariate analysis.

considered age as a limiting factor in prescribing thiopurines (Fig. 2).

\section{Discussion}

This study identified gastroenterologists preference and risk-perception with respect to prescribing various medication classes versus colectomy in elderly patients with steroid-dependent ulcerative colitis. First, vedolizumab was the most preferred treatment option for elderly patients with steroid-dependent ulcerative colitis. Second, both older age and the presence of comorbidity were independently associated with selection of vedolizumab and avoidance of immunomodulators and anti-TNF. In contrast, the presence of comorbidity rather than age-alone was the most important factor in recommending colectomy for steroid-dependent ulcerative colitis. Third, geographical heterogeneity was identified in the selection of treatment.

The ECCO consensus guidelines [22] recommended that steroid-dependent patients with moderate-to-severe ulcerative colitis be treated with a thiopurine, anti-TNF (preferably in combination with a thiopurine), or vedolizumab. Our survey identified that vedolizumab ranked first among these options. Older age, comorbidity, practicing in North America and Oceania, greater experience in managing IBD, and having a perceived age limit to prescribing thiopurines, were independent factors that were significantly associated with selecting vedolizumab as the preferred treatment. In clinical trials for infliximab [23] and golimumab [24], no upper age limit was set. For certolizumab [25], adalimumab [26], and vedolizumab [7], the upper age limits were 65,75 , and 80 years, respectively. Commencing thiopurines or anti-TNF agents in elderly patients is a challenge for physicians because of safety concerns for these medications. In a recent meta-analysis, the absolute risk of lymphoma in IBD patients treated with thiopurines was highest in patients $>50$ years old (1.35 cases per patient-year, with a relative risk of 4.78 ) [27]. A nationwide cohort study from France [17] provided evidence that patients exposed to anti-TNF monotherapy, thiopurine monotherapy, or combination therapy, had increased risks of serious infections compared with patients unexposed to either drug. In particular, the absolute risks were 2 -fold to 3 -fold greater in patients $>65$ years compared with younger patients [17]. Another retrospective, multicenter study [28] found that initiation of thiopurine therapy $>60$ years was associated with a higher risk of thiopurine-related adverse events. The efficacy and safety of vedolizumab are established in younger patients [29] and in the elderly [30]. A recent retrospective study [31] that compared the safety and efficacy of anti-TNF and vedolizumab in patients with IBD $>60$ years of age showed that both biological agents were similarly effective and well tolerated in this group of patients. There are limited data on the safety of vedolizumab in subjects with comorbidity. However, through its targeted, gut selective mechanism of action, vedolizumab is a well tolerated alternative to systemic immunosuppression and as such should be an attractive option in the elderly and for patients with comorbidities.

In addition to older age, patient comorbidity was another independent factor associated with less frequent use of immunomodulators and anti-TNF. Few data are available on the effect of comorbidity on the use of immunomodulators in elderly IBD. A systematic review and meta-analysis [10] along with cohort studies $[5,32]$ reported lower utilization of conventional immunomodulators and biological therapies in elderly-onset IBD compared with young-onset IBD. It is uncertain if 


\section{Cumulative Percentages of Gastroenterologists who have age limits for prescribing} IM and Biological Agents

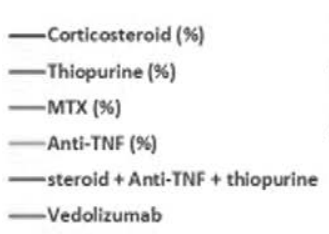

$\infty 0$
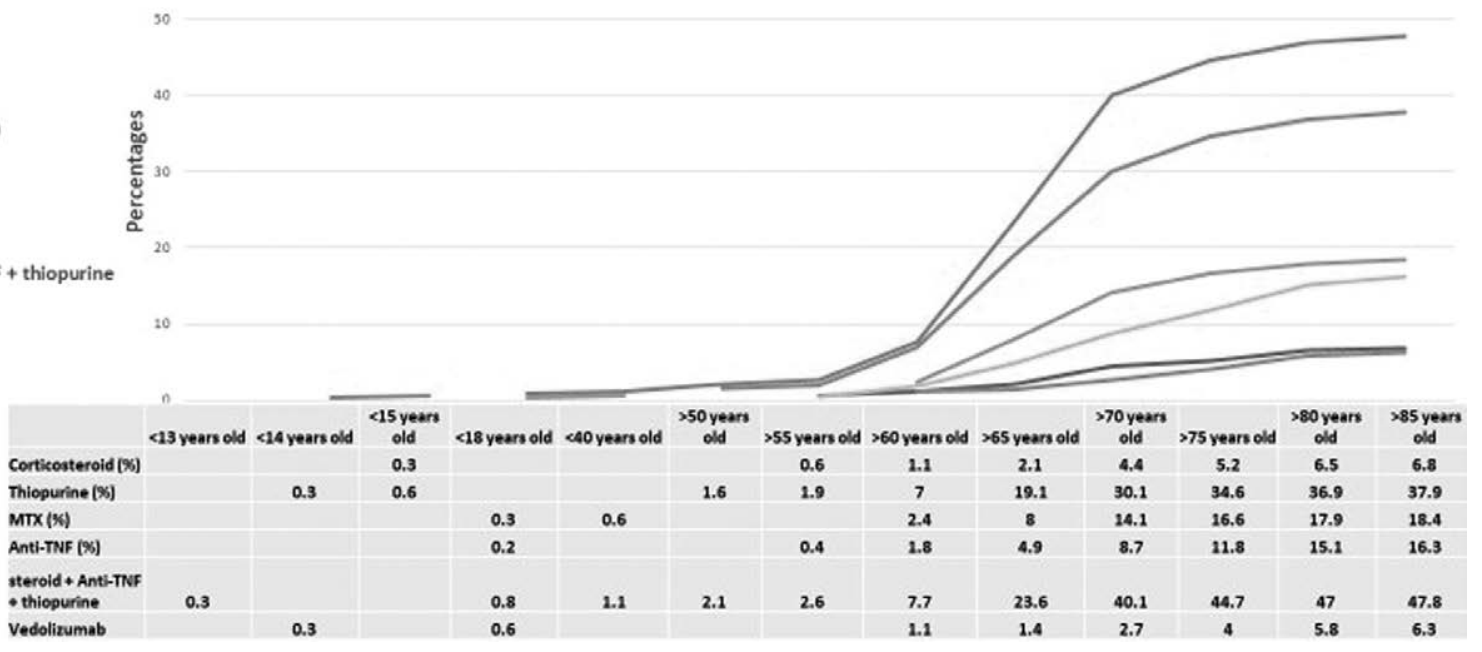

Fig. 2. Cumulative percentages of gastroenterologists who have age limits for prescribing immunomodulators and biological agents.

the underutilization of such therapies reflects the disease course of IBD [5,10,32], physicians' preference, or both. Kariyawasam et al. [33] previously reported in a comparative study of elderly-onset and young-onset IBD patients, that the Charlson's Comorbidity Index was shown to delay immunomodulator introduction in both Crohn's disease (hazard ratios: 0.86; 95\% CI, 0.79-0.95) and ulcerative colitis (hazard ratios: $0.81 ; 95 \% \mathrm{CI}, 0.71-0.92$ ) [33]. Lymphoma was regarded as the major contraindication to thiopurines in elderly patients. Following the diagnosis of a solid organ cancer, as many as 2-3 times more gastroenterologists would stop thiopurines $(65.1 \%)$ or anti-TNF agents $(57.4 \%)$ than vedolizumab $(20 \%$, $P \leq 0.0001)$. There was a surprisingly high proportion of respondents to this survey that did not avoid using antiTNF in the presence of demyelination, recent melanoma, or congestive heart failure.

The presence of comorbidity was the single most important factor in influencing the decision in recommending colectomy. Colectomy is generally viewed as being a curative option in ulcerative colitis, resulting in the cessation of all immunomodulator and biological drugs. However, this decision must be balanced against the increased rate of postoperative morbidity and mortality in operative subjects aged over 60 years with comorbidities [34]. Recent population-based studies demonstrated higher surgical rates in elderly-onset ulcerative colitis compared to young-onset ulcerative colitis patients $[5,35,36]$. The increased surgical rate was associated with very low frequency of immunomodulator use. Therefore, the decision to perform colectomy might have been driven by the treating physician's decision to select colectomy to avoid medical therapy, rather than the greater severity of ulcerative colitis.

Geographical heterogeneity was identified in the selection of immunomodulators and biological therapies. Physicians from Oceania and North America used vedolizumab significantly more frequently than other regions. European and North American gastroenterologists used immunomodulators less frequently compared with Asian countries. International variation in the treatment of patients with IBD is well-described [37]. Different healthcare systems, policies for medication reimbursement, and universal health insurance may influence the choice of medication prescription. For example, the Australian Pharmaceutical Benefits Scheme allows access to vedolizumab in the treatment of moderate-to-severe Crohn's disease and ulcerative colitis, even as the first-line biological agent, after failure of corticosteroids, mesalazine and an immunomodulator [38]. In Canada, vedolizumab is funded through public provincial drug plans for the treatment of IBD since 2017. Gastroenterologists from these countries, therefore, might select the newer biological agents that might be safer in the elderly. Gastroenterologists from South America, on the other hand, used anti-TNF more frequently due to the cost-benefit of infliximab biosimilar which has been available since 2015 .

The strength of this study was the recruitment of hundreds of gastroenterologists from various countries across the world to reflect the global practice pattern. It demonstrates that vedolizumab has gained widespread acceptance worldwide as the first-line therapy in moderate to severe ulcerative colitis in the elderly. This study has limitations. First, despite the large sample size of respondents, the absolute numbers of gastroenterologists from some individual countries were small. Therefore, we analysed practice across regions based on similar healthcare systems and patient demographics. This increased statistical power and the ability to identify geographical heterogeneity in medication prescribing practice. Second, there was no possible calculation of the response rate to the survey as the exact number of invitations was unknown. Third, selection bias was possible in respondents who are familiar with vedolizumab. However, some respondents responded with limited use of vedolizumab suggesting a wide cross-section of physicians who responded to the survey. We also controlled for expertise in IBD based on the average number of IBD patients that the respondents saw per week. Very few differences were identified based on high volume IBD practices versus low volume practices. Fourth, we focused 
on moderate-to-severe steroid-dependent ulcerative colitis in this survey. The pattern of use of therapies in paediatric subjects with IBD, and those with luminal Crohn's disease or perianal Crohn's disease will be the subject of future surveys. These data will likely differ from our current results. Last, we did not explore newer treatment options such as ustekinumab [8] and tofacitinib [9]. However, at the time of the survey, these agents were not yet available in most parts of the world and few respondents would have had ready access to these agents.

In conclusion, vedolizumab is the preferred firstline agent in the treatment of elderly IBD patients with steroid-dependent moderate-to-severe ulcerative colitis worldwide. Both old age and presence of comorbidity are the main factors that physicians consider when choosing between immunomodulators, anti-TNF, vedolizumab, and surgery. In elderly IBD patients, the presence of opportunistic infection and lymphoma were the main barriers in prescribing biological therapies and thiopurines, respectively. More data on the efficacy and safety of vedolizumab in the elderly IBD is needed to endorse it as the preferred first-line therapy in this group of patients.

\section{Acknowledgements}

The design, data analysis and manuscript writing were performed independently by the authors without external funding.

\section{Conflicts of interest}

There are no conflicts of interest.

W.C.: Guarantor of the article. W.C. and V.C.K. contributed equally to study design, data acquisition, analysis, and wrote and revised the manuscript. S.K. and H.H.S. contributed to data acquisition and manuscript revision. A.P., S.P., F.H.M., N.D., M.F., and R.W.L. contributed to study design, manuscript revision, and intellectual content. All authors approved the final version of the manuscript.

European Crohn's and Colitis Organisation Congress 2018 - Poster presentation.

\section{References}

1 Ng SC, Shi HY, Hamidi N, Underwood FE, Tang W, Benchimol El, et al. Worldwide incidence and prevalence of inflammatory bowel disease in the $21^{\text {st }}$ century: a systematic review of population-based studies. Lancet 2018; 390:2769-2778.

2 Sturm A, Maaser C, Mendall M, Karagiannis D, Karatzas P, Ipenburg $\mathrm{N}$, et al. European Crohn's and Colitis Organisation Topical Review on IBD in the elderly. J Crohns Colitis 2017; 11:263-273.

3 Loftus CG, Loftus EV Jr, Harmsen WS, Zinsmeister AR, Tremaine WJ, Melton LJ $3^{\text {rd }}$, Sandborn WJ. Update on the incidence and prevalence of Crohn's disease and ulcerative colitis in Olmsted County, Minnesota, 1940-2000. Inflamm Bowel Dis 2007; 13:254-261.

4 Gisbert JP, Chaparro M. Systematic review with meta-analysis: inflammatory bowel disease in the elderly. Aliment Pharmacol Ther 2014; 39:459-477.

5 Everhov ÅH, Halfvarson J, Myrelid P, Sachs MC, Nordenvall C, Söderling $\mathrm{J}$, et al. Incidence and treatment of patients diagnosed with inflammatory bowel diseases at 60 years or older in Sweden. Gastroenterology 2018; 154:518-528.e15.

6 Liu J, Kariyawasam V, Borody T, Katelaris P, Chan W, James C, et al. High age-specific prevalence of inflammatory bowel disease amongst the elderly in the city of Canada Bay area, Sydney: a metropolitan, population-based study. Gut 2018;67:A35-A36.

7 Feagan BG, Rutgeerts P, Sands BE, Hanauer S, Colombel JF, Sandborn WJ, et al.; GEMINI 1 Study Group. Vedolizumab as induction and maintenance therapy for ulcerative colitis. N Engl J Med 2013; 369:699-710.

8 Feagan BG, Sandborn WJ, Gasink C, Jacobstein D, Lang Y, Friedman JR, et al.; UNITI-IM-UNITI Study Group. Ustekinumab as Induction and Maintenance Therapy for Crohn's Disease. N Engl J Med 2016; 375:1946-1960.

9 Sandborn WJ, Su C, Sands BE, D'Haens GR, Vermeire S, Schreiber $\mathrm{S}$, et al.; OCTAVE Induction 1, OCTAVE Induction 2, and OCTAVE Sustain Investigators. Tofacitinib as induction and maintenance therapy for ulcerative Colitis. N Engl J Med 2017; 376:1723-1736.

10 Ananthakrishnan AN, Shi HY, Tang W, Law CC, Sung JJ, Chan FK, $\mathrm{Ng}$ SC. Systematic review and meta-analysis: phenotype and clinical outcomes of older-onset inflammatory bowel disease. J Crohns Colitis 2016; 10:1224-1236.

11 Juneja M, Baidoo L, Schwartz MB, Barrie A $3^{\text {rd }}$, Regueiro M, Dunn M, Binion DG. Geriatric inflammatory bowel disease: phenotypic presentation, treatment patterns, nutritional status, outcomes, and comorbidity. Dig Dis Sci 2012; 57:2408-2415.

12 Román AL, Muñoz F. Comorbidity in inflammatory bowel disease. World J Gastroenterol 2011; 17:2723-2733.

13 Parian A, Ha CY. Older age and steroid use are associated with increasing polypharmacy and potential medication interactions among patients with inflammatory bowel disease. Inflamm Bowel Dis 2015; 21:1392-1400.

14 Lopez A, Billioud V, Peyrin-Biroulet C, Peyrin-Biroulet L. Adherence to anti-TNF therapy in inflammatory bowel diseases: a systematic review. Inflamm Bowel Dis 2013; 19:1528-1533.

15 Lemaitre M, Kirchgesner J, Rudnichi A, Carrat F, Zureik M, Carbonnel F, Dray-Spira R. Association between use of thiopurines or tumor necrosis factor antagonists alone or in combination and risk of lymphoma in patients with inflammatory bowel disease. JAMA 2017; 318:1679-1686.

16 Naganuma M, Kunisaki R, Yoshimura N, Takeuchi Y, Watanabe M. A prospective analysis of the incidence of and risk factors for opportunistic infections in patients with inflammatory bowel disease. $J$ Gastroenterol 2013; 48:595-600.

17 Kirchgesner J, Lemaitre M, Carrat F, Zureik M, Carbonnel F, DraySpira R. Risk of serious and opportunistic infections associated with treatment of inflammatory bowel diseases. Gastroenterology 2018; 155:337-346.e10.

18 Bonovas S, Fiorino G, Allocca M, Lytras T, Nikolopoulos GK, PeyrinBiroulet L, Danese S. Biologic therapies and risk of infection and malignancy in patients with inflammatory bowel disease: a systematic review and network meta-analysis. Clin Gastroenterol Hepatol 2016; 14:1385-1397.e10.

19 Peyrin-Biroulet L, Khosrotehrani K, Carrat F, Bouvier AM, Chevaux JB, Simon T, et al.; Cesame Study Group. Increased risk for nonmelanoma skin cancers in patients who receive thiopurines for inflammatory bowel disease. Gastroenterology 2011; 141:1621-28.e1.

20 Abbas AM, Almukhtar RM, Loftus EV Jr, Lichtenstein GR, Khan N. Risk of melanoma and non-melanoma skin cancer in ulcerative colitis patients treated with thiopurines: a nationwide retrospective cohort. Am J Gastroenterol 2014; 109:1781-1793.

21 Chan W, Shim HH, Ng SC, Liu J, Inglis C, Greveson K, et al.; AsiaPacific Crohn's and Colitis Epidemiologic Study (ACCESS) Study Group. A global survey of gastroenterologists' travel advice to patients with inflammatory bowel disease on immunosuppressive agents and management of those visiting tuberculosis-endemic areas. J Crohns Colitis 2018; 12:1261-1269.

22 Harbord M, Eliakim R, Bettenworth D, Karmiris K, Katsanos K, Kopylov U, et al.; European Crohn's and Colitis Organisation. Third European Evidence-based Consensus on Diagnosis and Management of Ulcerative Colitis. Part 2: current management. J Crohns Colitis 2017; 11:769-784.

23 Rutgeerts P, Sandborn WJ, Feagan BG, Reinisch W, Olson A, Johanns $\mathrm{J}$, et al. Infliximab for induction and maintenance therapy for ulcerative colitis. N Engl J Med 2005; 353:2462-2476.

24 Sandborn WJ, Feagan BG, Marano C, Zhang H, Strauss R, Johanns J, et al.; PURSUIT-Maintenance Study Group. Subcutaneous golimumab maintains clinical response in patients with moderate-to-severe ulcerative colitis. Gastroenterology 2014; 146:96-109.e1.

25 Sandborn WJ, Feagan BG, Stoinov S, Honiball PJ, Rutgeerts P, Mason D, et al.; PRECISE 1 Study Investigators. Certolizumab pegol for the treatment of Crohn's disease. N Engl J Med 2007; 357:228-238.

26 Sandborn WJ, van Assche G, Reinisch W, Colombel JF, D'Haens G, Wolf DC, et al. Adalimumab induces and maintains clinical remission in 
patients with moderate-to-severe ulcerative colitis. Gastroenterology 2012; 142:257-65.e1.

27 Kotlyar DS, Lewis JD, Beaugerie L, Tierney A, Brensinger CM, Gisbert $\mathrm{JP}$, et al. Risk of lymphoma in patients with inflammatory bowel disease treated with azathioprine and 6-mercaptopurine: a meta-analysis. Clin Gastroenterol Hepatol 2015; 13:847-58.e4; quiz e48.

28 Calafat M, Mañosa M, Cañete F, Ricart E, Iglesias E, Calvo M, et al.; ENEIDA registry of GETECCU. Increased risk of thiopurine-related adverse events in elderly patients with IBD. Aliment Pharmacol Ther 2019; 50:780-788.

29 Colombel JF, Sands BE, Rutgeerts P, Sandborn W, Danese S, D'Haens G, et al. The safety of vedolizumab for ulcerative colitis and Crohn's disease. Gut 2017; 66:839-851.

30 Schreiber S, Dignass A, Peyrin-Biroulet L, Hather G, Demuth D, Mosli $\mathrm{M}$, et al. Systematic review with meta-analysis: real-world effectiveness and safety of vedolizumab in patients with inflammatory bowel disease. J Gastroenterol 2018; 53:1048-1064.

31 Adar T, Faleck D, Sasidharan S, Cushing K, Borren NZ, Nalagatla N, et al. Comparative safety and effectiveness of tumor necrosis factor $\alpha$ antagonists and vedolizumab in elderly IBD patients: a multicentre study. Aliment Pharmacol Ther 2019; 49:873-879.

32 Jeuring SF, van den Heuvel TR, Zeegers MP, Hameeteman WH, Romberg-Camps MJ, Oostenbrug LE, et al. Epidemiology and long-term outcome of inflammatory bowel disease diagnosed at elderly age-an increasing distinct entity? Inflamm Bowel Dis 2016; 22:1425-1434.

33 Kariyawasam VC, Kim S, Mourad FH, Selinger CP, Katelaris PH, Brian Jones D, et al. Comorbidities rather than age are associated with the use of immunomodulators in elderly-onset inflammatory bowel disease. Inflamm Bowel Dis 2019; 25:1390-1398.

34 Sacleux SC, Sarter H, Fumery M, Charpentier C, Guillon-Dellac N, Coevoet $\mathrm{H}$, et al.; EPIMAD Group. Post-operative complications in elderly onset inflammatory bowel disease: a population-based study. Aliment Pharmacol Ther 2018; 47:1652-1660.

35 Nguyen GC, Bernstein CN, Benchimol El. Risk of surgery and mortality in elderly-onset inflammatory bowel disease: a population-based Cohort Study. Inflamm Bowel Dis 2017; 23:218-223.

36 Mañosa M, Calafat M, de Francisco R, García C, Casanova MJ, Huelín P, et al.; GETECCU. Phenotype and natural history of elderly onset inflammatory bowel disease: a multicentre, case-control study. Aliment Pharmacol Ther 2018; 47:605-614.

37 Benchimol El, Cook SF, Erichsen R, Long MD, Bernstein CN, Wong $\mathrm{J}$, et al. International variation in medication prescription rates among elderly patients with inflammatory bowel disease. J Crohns Colitis 2013; 7:878-889.

38 Vedolizumab, The Pharmaceutical Benefits Scheme, the Department of Health, Australian Government. https://www.pbs.gov.au/medicine/ item/10384M-10390W-10398G-10415E. [Accessed 26 July 2019] 